\title{
Streptococcal Pharyngitis
}

National Cancer Institute

\section{Source}

National Cancer Institute. Streptococcal Pharyngitis. NCI Thesaurus. Code C116003.

Inflammation of the throat due to Streptococcus pyogenes. 